American Journal of Applied Sciences 5 (9): 1144-1148, 2008

ISSN 1546-9239

(C) 2008 Science Publications

\title{
Quantum Hadamard Gate Implementation Using Planar Lightwave Circuit and Photonic Crystal Structures
}

\author{
Shamsolah Salemian and Shahram Mohammadnejad \\ Department of Electrical Engineering, \\ Iran University of Science and Technology (IUST), \\ Narmak, 16846, Tehran, Iran
}

\begin{abstract}
An all optical method has been proposed for quantum Hadamard gate implementation. This quantum gate was realized by using Y-junction beam splitter. Normal modes in waveguides have been used as quantum bit. This all optical gate can be used in quantum computation and communication. The proposed Hadamard gate has potential of being more compact and easily realized compared to other optical implementations. By using planar lightwave circuit in implementation, the width of Yjunction input waveguide, width of each branch, angle of bend and length of bend were obtained 1 micron, 0.75 micron, $45^{\circ}$ and 0.75 micron, respectively. By using planar lightwave circuit in implementation, the radius of large air holes and the radius of small holes were obtained 0.2 ands 0.1 micron, respectively. The index of substrate was 1.325 . Implementation based on photonic crystal structures and planar lightwave circuit technology was used in integrated optics.
\end{abstract}

Key words: Quantum gate, quantum communication, quantum computation, qubit, integrated optics

\section{INTRODUCTION}

Quantum gates are important part of quantum communication and computation. Many researchers work on theories of quantum computation, such as entanglement, quantum decoherernce, quantum cryptography and quantum error correction. Now Quantum computation and communication move closer to being a reality ${ }^{[1,2,3]}$. For transition from theory to applications, we need to realize quantum computer by using the implementation of quantum gates. Standard versions of Planar Lightwave Circuit (PLC) technology are well documented and have been practiced since late 1980 's. They combine significant features of optical fiber and integrated circuit technologies. Basically, light-guiding channels, similar in function to optical fibers, are defined on a silicon platform. These are fabricated by depositing sequential glass layers onto silicon wafers. Typically, an intermediate "core" layer with an elevated refractive index is patterned using photolithography and dry-etching. This patterned structure becomes the light-guiding channel. This baseline technology is well suited to the fabrication of passive devices such as couplers, which depend on the proximity, controlled spacing and path lengths of parallel waveguides.
The silicon chip provides a robust support for the waveguides. Such PLC components are interfaced to fiber optic networks via edge attached fibers. This technology supports complex and versatile photonic integration. Implementation of quantum gates by using all optical circuits is very important in all optical communication networks. Since the trend for photonics integration is toward smaller and more densely-packed components, it is necessary to be able to model these small components perfectly ${ }^{[4,5,6]}$.

Photonic crystal structures are another technology that used for realization of integrated optics component, such as switches, couplers and multiplexer.

\section{MATERIALS AND METHODS}

Proposed model for Hadamard gate: Qubits can be realized by two normal modes of dual-mode waveguides, such as the zero logical state $|0\rangle$ encoded into one normal mode, $\mathrm{TE}_{0}$ and the logical one $|1\rangle$ given by other orthogonal normal mode, TE1.

A qubit's state space consists of all superpositions of the basic normal modes $|0\rangle$ and $|1\rangle$. Hadamard gate has one input and one output. Operation of this gate has been shown below,

Corresponding Author: Shamsolah Salemian, Department of Electrical Engineering, Iran University of Science and Technology (IUST), Narmak, 16846, Tehran, Iran 


$$
H=\left\{\begin{array}{l}
\left|0>\rightarrow \frac{1}{\sqrt{2}}\right| 0>+\frac{1}{\sqrt{2}} \mid 1> \\
\left|1>\rightarrow \frac{1}{\sqrt{2}}\right| 0>-\frac{1}{\sqrt{2}} \mid 1>
\end{array}\right.
$$

An integrated optics beam splitter will be proposed for realization of this gate. Beam splitter is a basic element of many optical fiber communication systems often providing a Y-junction by which signals from separate sources can be combined, or the received power can be divided between two or more channels ${ }^{[7]}$. A passive Y-junction beam splitter is shown in Fig. 1. Unfortunately, the power transmission through such a splitter decreases sharply with increasing half angle $\theta$, the power being radiated into substrate. The passive $\mathrm{Y}$-junction beam splitter finds application where equal power division of incident beam is required.

We consider waveguide branch that support the two lowest order normal modes $\mathrm{TE}_{0}$ and $\mathrm{TE}_{1}$ $(|0\rangle$ and $|1\rangle)$. Electric-field profiles of these modes are shown in Fig. 2.

Power transfer between these modes in the branch is described by the coupled mode equations. Because of linearity of these equations and superposition properties of Maxwell's equations, the solution can be obtained for each local normal mode independently and superimpose these solutions at the output side of the branch, at large branch arm separation. Normal modes $\mathrm{TE}_{0}$ and $\mathrm{TE}_{1}$ $(|0\rangle$ and $|1\rangle)$ with equal powers and phases are applied on an ideal power dividing branch. Considering each normal mode independently, the output mode amplitudes in branch 1 are same and have value equal to $\frac{1}{\sqrt{2}}$, but in branch 2 , output amplitudes of $\mathrm{TE}_{0}$ and $\mathrm{TE}_{1}$ mode are $\frac{1}{\sqrt{2}}$ and $-\frac{1}{\sqrt{2}}$, respectively.

Therefore, the output of branch 1 is the same as output of Hadamard gate when its input is $|0\rangle$ and the output of branch 2 is the same as output of Hadamard gate when its input is $|1\rangle$. The relation between output and input of this element is,

$$
\left[\begin{array}{l}
\psi_{1} \\
\psi_{2}
\end{array}\right]_{\text {out }}=\frac{1}{\sqrt{2}}\left[\begin{array}{rr}
1 & 1 \\
1 & -1
\end{array}\right]\left[\begin{array}{l}
|0\rangle \\
|1\rangle
\end{array}\right]_{\text {in }}
$$

Output of this element is exactly same as Hadamard gate.

Planar Lightwave Circuit: Silicon on insulator waveguide technology is an important contender among

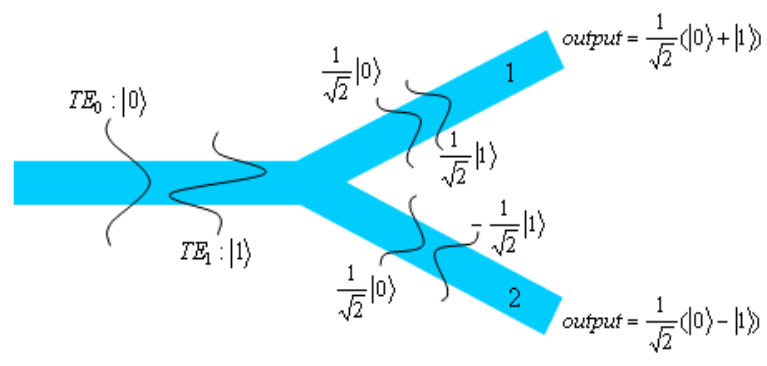

Fig. 1: Y-junction beam splitter as a Hadamard gate

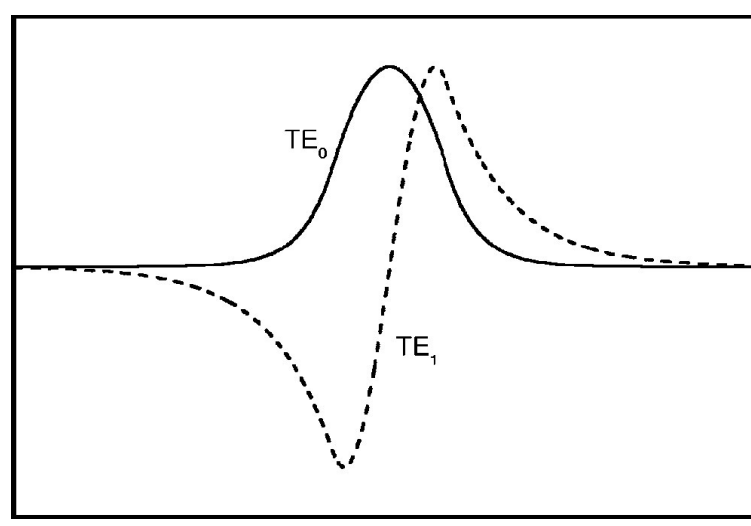

Fig. 2: Electric-field profiles for modes $\mathrm{TE}_{0}$ (the symmetric mode) and $\mathrm{TE}_{1}$ (the antisymmetric mode)

the Planar Lightwave Circuit (PLC) technologies to overcome the limitations of the current silica-on-silicon technology. Silica-on-Silicon suffers from physical constraints (index contrast) that prevent a further size reduction and therefore higher integration of passive optical components. The inherent high index contrast of the Silicon-Silicon dioxide material system leads to strong optical confinement, which in turn permits a strong miniat urization of waveguides, bends and devices $^{[8]}$.

Photonic crystal structures: Photonic crystal devices have the potential to be very small and designing devices based on photonic crystals offers the ability to engineer the electromagnetic fields in microscopic detail. Some of this potential has begun to be realized in the design and demonstration of very small resonant cavities, small turning radius waveguide bends and wide angle Y-branches leading to the possibility of dense device integration. There is much research to be done, however, before photonic crystal device technology is mature ${ }^{[9]}$. 


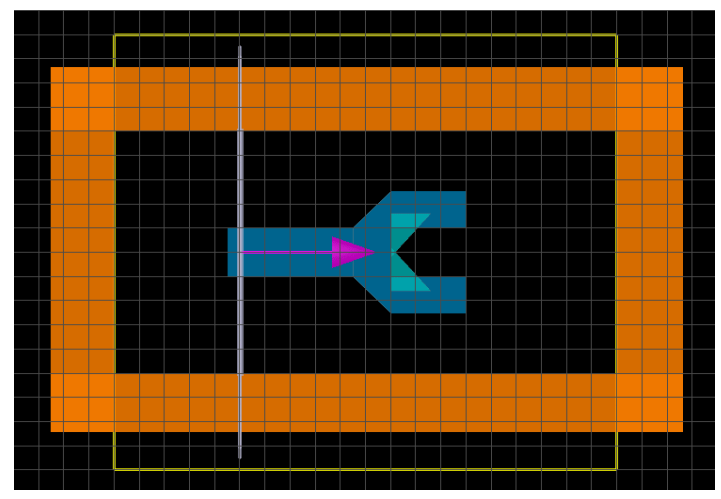

Fig. 3: Hadamard gate implementation using planar light wave circuit

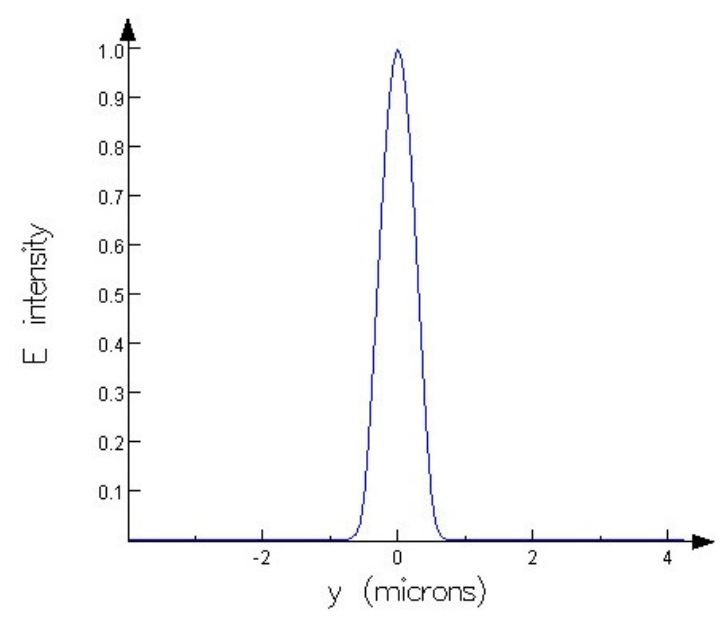

Fig. 4: Electric field profile of source 1

\section{RESULTS AND DISCUSSION}

Hadamard Gate Implementation Using Planar Lightwave Circuit:In this section, hadamard gate will be designed by using Silicon-Silicon dioxide planar light wave technique. The designed hadamard gate has been shown in Fig. 3. The width of input waveguide of Y-junction is 1 micron and width of each branch is equal to 0.75 micron. The angle of bend is $45^{\circ}$ and its length is 0.75 micron. Two sources have been used in simulation. Wavelengths of these sources are 1.55 micron and their polarization is TE. Two normal modes are selected for sources. Electric field profiles of these modes have been shown in Fig. 4 and 5 .

In the first step of simulation, each source is applied separately. The output power of each branch for each source is equal to half of input power. The results of simulation for source 1 and source 2 have been shown in Fig. 6 and 7, respectively.

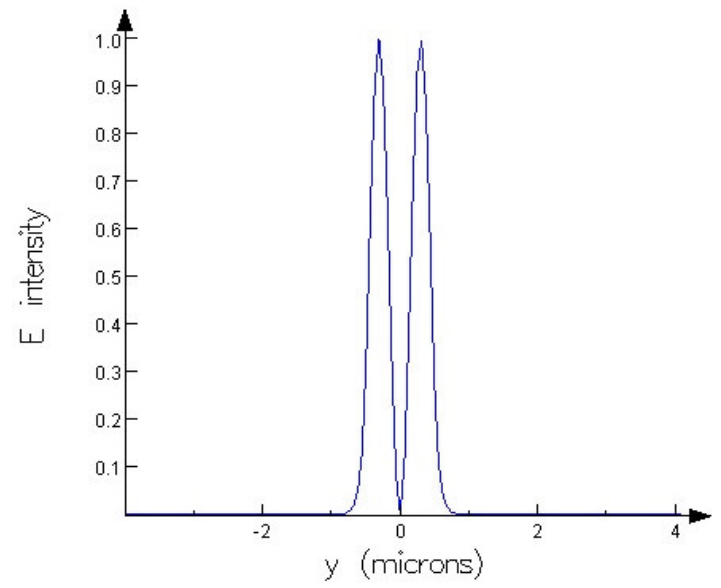

Fig. 5: Electric field profile of source 2

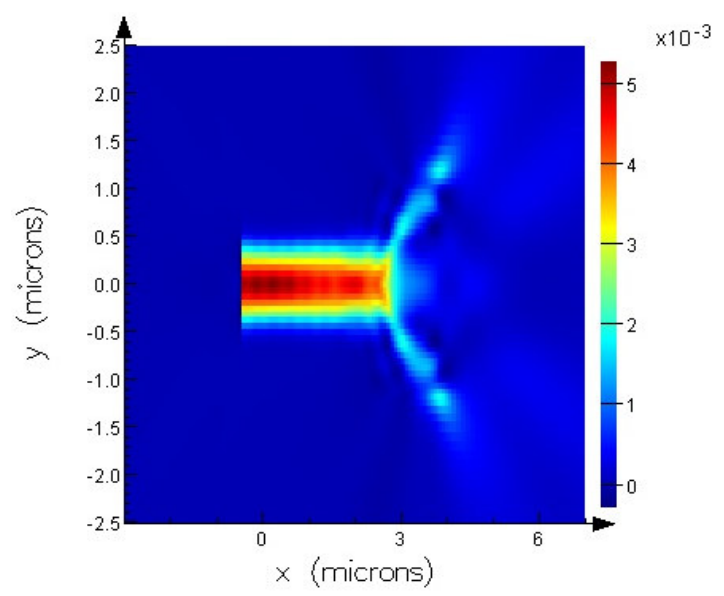

Fig. 6: Intensity of power vs. position for input source 1

If two sources are applied simultaneously, according to Eq. 1 and Fig. 1 we expect that power of source 2 subtract from power of source 1 in branch 2 and total power in this branch decrease. Figure 8 shows this result.

Hadamard gate implementation using photonic crystal structures: The designed Hadamard gate based on photonic crystal structure has been shown in Fig. 9. In this scheme the large air holes have radius equal to 0.2 micron and radius of small holes is 0.1 micron. Index of substrate is 1.325 . The defined sources in previous section are used for the present case.

Same as previous section, at first each source is applied separately. We expect that the output power of each branch for each source is equal to half of input power. The results of simulation for source 1 and source 2 have been shown in Fig. 10 and 11, respectively. 


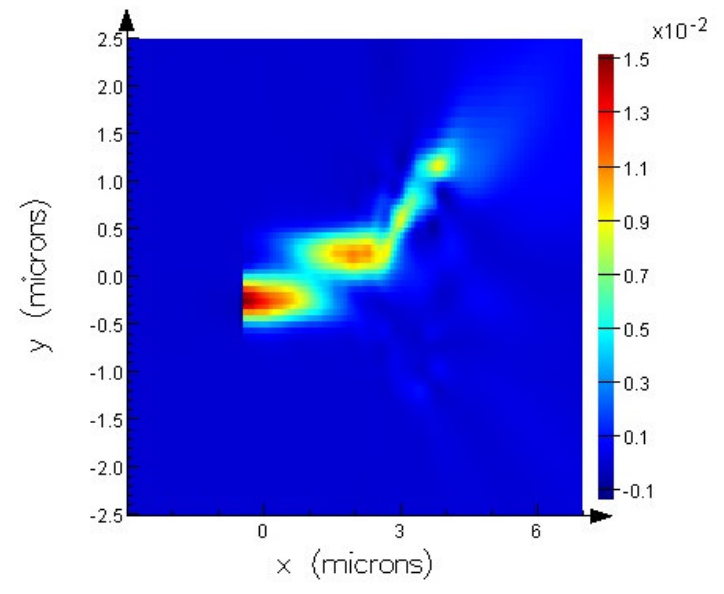

Fig. 8: Intensity of power vs. position for appling two sources simultanousely

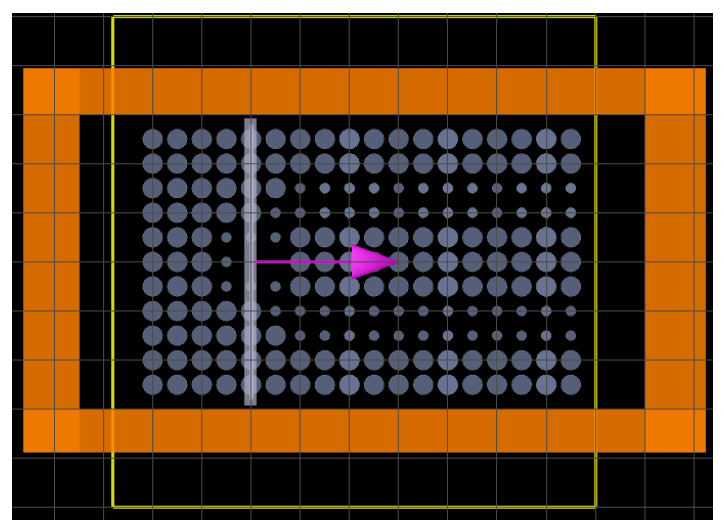

Fig. 9: Hadamard gate implementation using photonic crystal structure

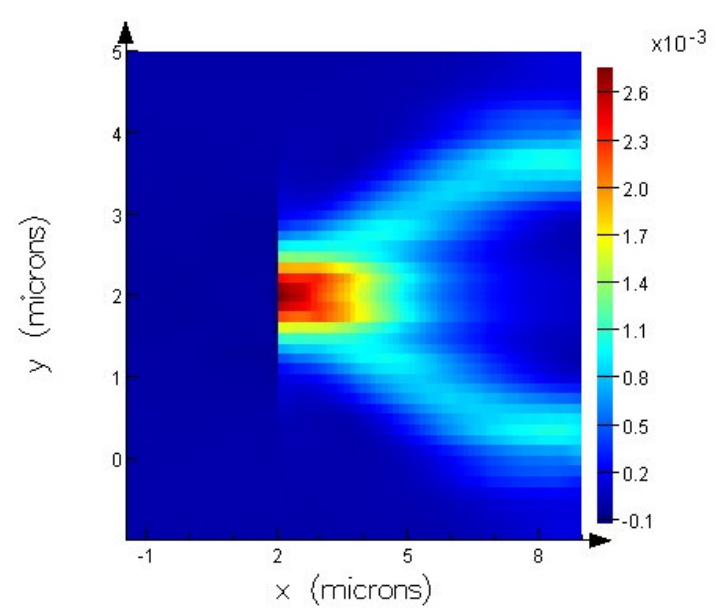

Fig. 10: Intensity of power vs. position for input source 1

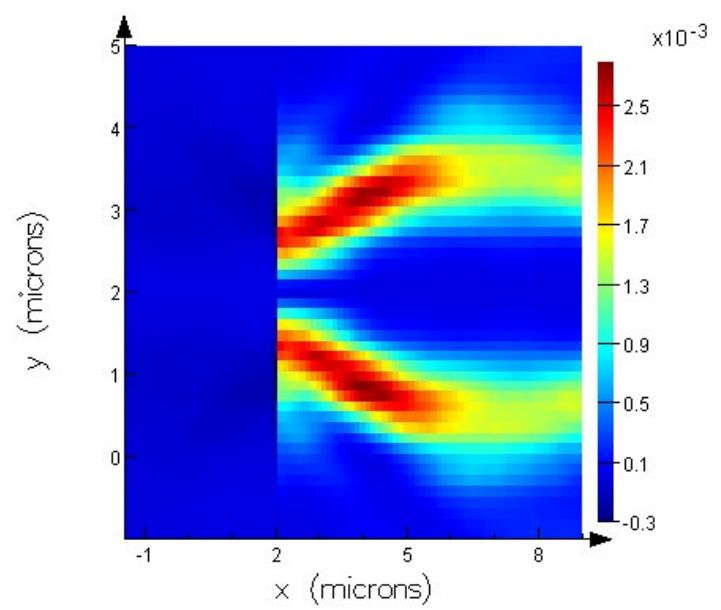

Fig. 11: Intensity of power vs. position for input source 2

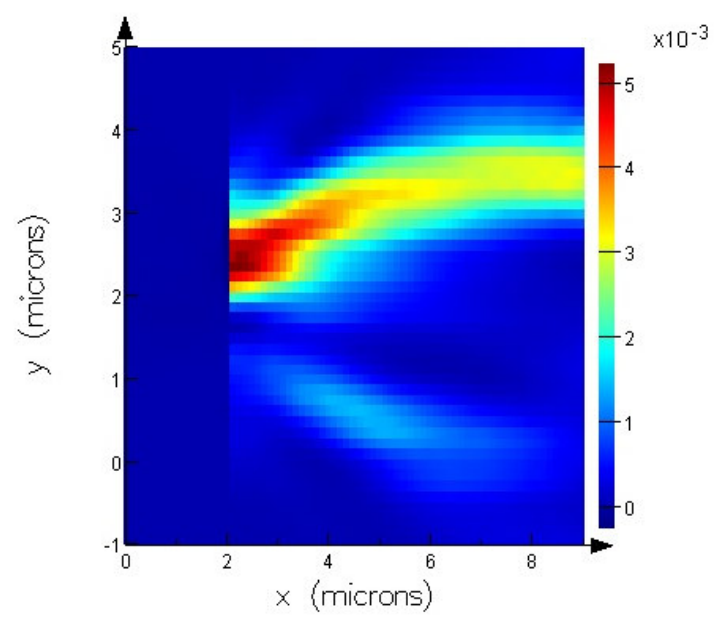

Fig. 12: Intensity of power vs. position for appling two sources simultanousely

If two sources are applied simultaneously, same as previous section, we expect that power of source 2 subtract from power of source 1 in branch 2 and total power in this branch decrease. Figure 12 shows this result.

\section{CONCLUSION}

In conclusion, we proposed two all optical methods for implementation of quantum Hadamard gate. By using planar lightwave technology and photonic crystal waveguide, Hadamard gate is feasible. These technologies will be used in new integrated optics devices and help us to reduce size of optical devices. For example, in this paper, the size of implemented 
Hadamerd gate is less than 6 micron. By using a Y-junction beam splitter, a fully optical model to perform quantum Hadamard gate as a universal gate for quantum communication and computation was proposed.

\section{REFERENCES}

1. Benenti, G., G. Casati and G. Strini, 2004. Principles of quantum computation and information. Basic concepts. World Scientific, Singapore, Vol. I.

2. Benenti, G. and G. Strini, 2007. A bird's eye view of quantum computers. Quantum Biosyst., 1 (1): 21-30.

3. Nielsen, M.A. and I.L. Chuang, 2000. Quantum computation and quantum information. Cambridge University Press, Cambridge.
4. Okamoto, K., 1999. Recent progress of integrated optics planar lightwave circuits. Optical and Quantum Electronics. Springer Netherlands, 31 (2): 107-129.

5. Doerr, C.R., 2006. Advances in Silica Planar Lightwave Circuits. J. Lightwave Technol., 24 (12): 4763-4789.

6. Suzuki, S. and A. Sugita, 2005. Recent Progress in Silica-based PlanarLightwave Circuits (PLCs). NTT Tech. Rev., 3 (7): 12-16.

7. Tamir, T., 1988. Guided-wave Optoelectronics. Springer-Veriag, New York, pp: 89-125.

8. Zimmermann, L., 2006. Silicon-on-Insulator based micro-optical integration platform. IEEE Lasers and Electro-Optics Society LEOS-Hong Kong.

9. O'Brien, J., Min-Hsiung Shih and T. Yang, 2006. Photonic Crystal Devices. University of Southern California, Los Angeles, CA 90089-0271. 